\title{
No-reference blur image quality assessment based on Simulated Annealing and General Regression Neural Network
}

\author{
Zhongzhong LIU ${ }^{1, a}$, Tao CHENG ${ }^{2, b}$ * \\ ${ }^{1}$ The College of Mechatronics and Control Engineering, Shenzhen University, Shenzhen, P. R. \\ China, 518060 \\ 2 The College of Mechatronics and Control Engineering (The College of Urban Rail Transit), \\ Shenzhen University, Shenzhen, P. R. China, 518060 \\ akyle_lz@163.com, bchengtao@szu.edu.cn
}

\begin{abstract}
Keywords: General Regression Neural Network; no-reference; image quality assessment; Simulated Annealing

Abstract. In order to improve the accuracy and efficiency of no-reference blur image quality assessment based on General Regression Neural Network. We choose Simulated Annealing algorithm to optimize the method. Using LIVE (Laboratory for Image \& Video Engineering) database as the initial study database. 174 images from LIVE database are assigned randomly to two groups. Phase-matched images generated by phase transformation. We can get Gray Level Co-occurrence Matrix form phase-matched images. Then, get the energy, Entropy, correlation, contrast and homogeneity of these five characteristics indexes. Using the above indicators as input data and using Difference Mean Opinion Score as output data. Training neural network model on this way. In order to improve the accuracy and efficiency, using the Simulated Annealing algorithm to find the optimal smoothing factor parameter. Finally, spearman correlation coefficient of objective and subjective data is 0.9319 . Pearson correlation coefficient of objective and subjective data is 0.9328 . The results show that, this algorithm fits Difference Mean Opinion Score well. It predict better on image quality assessment.
\end{abstract}

\section{Introduction}

People view digital images on social networks every day. Clear digital images are more likely to be accepted by human. If the images are not clear, then humans may misconceived by images. In the industrial field, image processing is widely used in pharmaceutical, semiconductor, printing, automotive components, monitoring, safety and food industries. Image quality directly affects the quality of product. Therefore, the study of image quality assessment method is very important.

Image quality assessment can be divided into subjective assessment and objective assessment. Subjective image quality assessment depends on one's subjective feelings. Difference Mean Opinion Score was given based on the subjective feelings of digital image. Objective image quality assessment is based on algorithm model. Due to the subjective image quality assessment consuming large human and material resources. Subjective image quality assessment can not be in real-time. Objective image quality assessment method is mainly used for practical applications. Objective image quality assessment can be divided into full reference image quality assessment, part reference image quality assessment and no reference image quality assessment. No-reference image quality assessment method does not need to extract original image data for assessment. No-reference image quality assessment methods is more flexibility and wider range of applications than reference image quality assessment methods ${ }^{[1]}$.

In recent years, no-reference image quality assessment study is very popular with researchers. Zhengyi Xiao studys image quality assessment basis on noise detection ${ }^{[2]}$. Yu Shao apply the relationship between tensor eigenvalues to measure the level of blurring image and image noise. He combines the two factors to obtain a comprehensive assessment model ${ }^{[3]}$. Yusai Zhang use edge amplitude, length, brightness, background activity and other visual sensitivity characteristics to achieve no-reference image quality assessment ${ }^{[4]}$. Ying Yin predict blurred image quality scores by phase transformation, Gray Level Co-occurrence Matrix and generalized regression neural networks ${ }^{[5]}$. The 
authors studied generalized neural network no-reference image quality assessment algorithms and Simulated Annealing algorithm. Proposed a no-reference blur image quality assessment algorithm based on Simulated Annealing and General Regression Neural Network. The results show that, this algorithm fits Difference Mean Opinion Score well. It performs better on image quality assessment.

\section{General Regression Neural Network based on Simulated Annealing}

GRNN model. GRNN (Generalized Regression Neural Network) is a branch of RBFNN (Radial Basis Function Neural Network). It is different from interpolation and fitting, when gets the relationship between data method. It amend the network by computing and samples, without recalculated parameters. Compared with BPNN (Back Propagation Neural Network) GRNN is better than BPNN on approximation ability, classification ability and learning speed. It can handle unstable data. It predicts will, even the sample data is short.

GRNN is a new neural network algorithm which was first proposed in 1991 by Donald Specht. The theory is based on non-linear regression analysis. It works from the idea of probability theory. When used as a function approximation, network output is a regression function of network input. When used as a classification, it is the posterior probability.

Suppose, random variable $x$ and random variable $y$. Their joint probability density function is $f(x, y)$. Variable $x$ observations value is $X$. Variable $y$ return equivalent to $x$. As shown in the following equations (1).

$$
\hat{y}=E(y \mid X)=\frac{\int_{-\infty}^{+\infty} y f(X, y) d y}{\int_{-\infty}^{+\infty} f(X, y) d y}
$$

GRNN consists of input layer, model layer and summation layer and output layer. Its network structure is shown in figure 1 below.

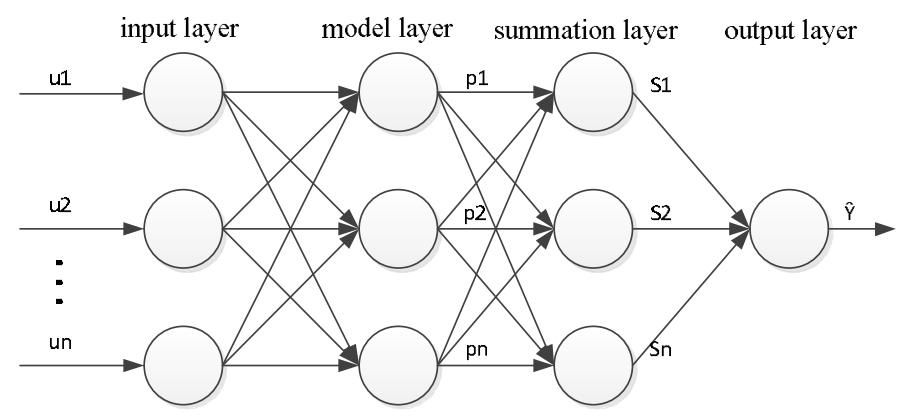

Fig.1 General Regression Neural Network network topology

The most distinguishing feature of GRNN is the basis function of pattern layer neurons. The activation function using radial basis function. Basis functions respond to the input signal. When the input signal is closer to the middle of the basis function of scope, pattern layer neural neurons will produce greater output. So the GRNN has local approximation ability.

In MATLAB (Matrix Laboratory), the GRNN model structure is simple to use. It only need to set up a smoothing factor parameter spread. Maximize the reduction influence of human factors to model. It reduces the network structure design. At present, the selection of parameters are mainly adopts manual adjustment method. This method is poor precision and low efficiency. Mathematically speaking, the determination of spread is an optimization problem. In the interval $[0,2]$ to find an optimal parameters. Make the output value of the training samples and the actual value has the minimum mean square error. Based on the Simulated Annealing algorithm optimize the GRNN model, and through MATLAB implementation.

The GRNN model based on Simulated Annealing optimization. Simulated Annealing algorithm is the result of statistical thermodynamics. Algorithm describes the simulation under the molten state object from cooling to ultimately achieve the physical process of crystalline state. 
Simulated Annealing algorithm takes advantage of the annealing process and melting object similarity this feature in the process of problem solving. It Simulated Annealing process object to complete solution of the problem ${ }^{[6]}$. According to the statistical thermodynamics, the condition of each molecule obey Gibbs distribution ${ }^{[7]}$. As shown in the following equations (2).

$$
\rho\left(r_{i}\right)=\frac{\exp \left(-\frac{E\left(r_{i}\right)}{k T}\right)}{\sum_{j=1}^{M} \exp \left(-\frac{E\left(r_{j}\right)}{k T}\right)}
$$

$r_{i}$ is the state of molecular $i . E\left(r_{i}\right)$ is the energy function of molecular $i . T$ represents temperature. $K$ is the Boltzmann constant.

This article uses the pearson correlation coefficient (Pearson Correlation Coefficient, CC) and spearman rank correlation coefficient (Spearman Rank - Order the Correlation Coefficient, SROCC) as the performance evaluation index. $\mathrm{CC}$ and $\mathrm{SROCC}$ value range of $[0,1]$, the greater the value the better the performance of algorithm. GRNN model uses the sum of SROCC and CC as the fitness function. The SA-GRNN process and MATLAB pseudo code is as follows.

(1) Setting up the Simulated Annealing calculation parameters.

$T=1$;

$\%$ The temperature of the system, the system should be in the condition of a high temperature

$T \_$min $=0.01$;

$\%$ The minimum temperature. If the temperature reaches the minimum, stop the search.

$r=0.95$;

$x 1=0.01$;

$\%$ Used to control the cooling speed

$x 2=0.01$;

\%current state

\%new state

(2) Sets the fitness function:

fitness $(i)=\operatorname{SROCC}(i)+C C(i) \quad \%$ fitness settings

(3) Import the samples and status value to the GRNN. Calculate the fitness value:

net=newgrnn $\left(\boldsymbol{P} \_\right.$net, $\left.\boldsymbol{T}_{-} \boldsymbol{n e t}, x 2\right) ; \%$ prediction score

net=newgrnn $\left(\boldsymbol{P} \_\right.$net, $\left.\boldsymbol{T}_{-} \boldsymbol{n e t}, x \mathbf{1}\right) ; \%$ prediction score

(4) Comparison before and after the state of the fitness value, the system cooling:

if $(d E>=0)$

$\%$ After moving to get optimal solution, mobile is accepted

else

\%accepted from $Y(i)$ to $Y(i+1)$

end

\%have a probability to accept from $Y(i)$ to move $Y(i+1)$

(5) After cooling, systems have smooth factor:

SPREAD $=x 1$

\%output smoothing factor

Image quality assessment based on SA algorithm and GRNN model process as shown in figure 2 . 


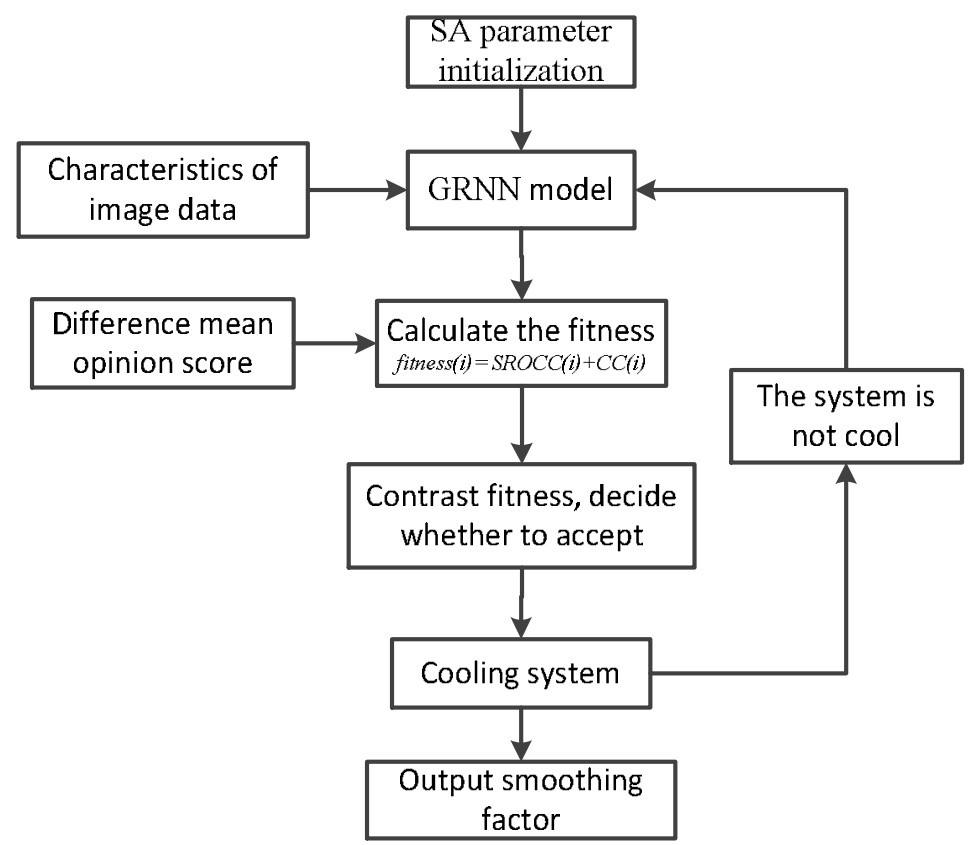

Fig.2 Flow chart of algorithm based on Simulated Annealing and General Regression Neural Network

\section{Image quality assessment based on SA - GRNN model}

SA - GRNN image quality assessment model. Morrone et al, the study found that the image of the mach band features highly in phase. Thus, this paper puts forward the principle of phase consistency $^{[8]}$. Phase consistency is a kind of important image features ${ }^{[9]}$. It can accurately describe the structure of the image, texture and edge information, image brightness or contrast has no effect on it. Liu and others use phase consistency puts forward a new method of image assessment ${ }^{[10]}$. As shown in the following equations (3).

$$
\mathrm{PC}(\mathrm{x})=\frac{\sum_{o}\left|E_{o}(x)-T_{o}\right|}{\sum_{o} \sum_{n} A_{n o}(x)+\varepsilon}
$$

$\varepsilon$ is a very small positive number. o Said direction. $T_{o}$ noise compensation factor. Figure 3-6 image is come from the university of Texas LIVE database Release $2^{[11,12]}$ "plane.bmp". The image of the ideal images, images and their corresponding fuzzy distortion in phase images respectively as shown in the figure below. From figure 3-6, we can found that, phase images well showed the structure of the image contour features. The blurred image in phase images are also reflects the image structure alteration. In phase images can be used as characteristic parameters extraction and image quality of important basis.
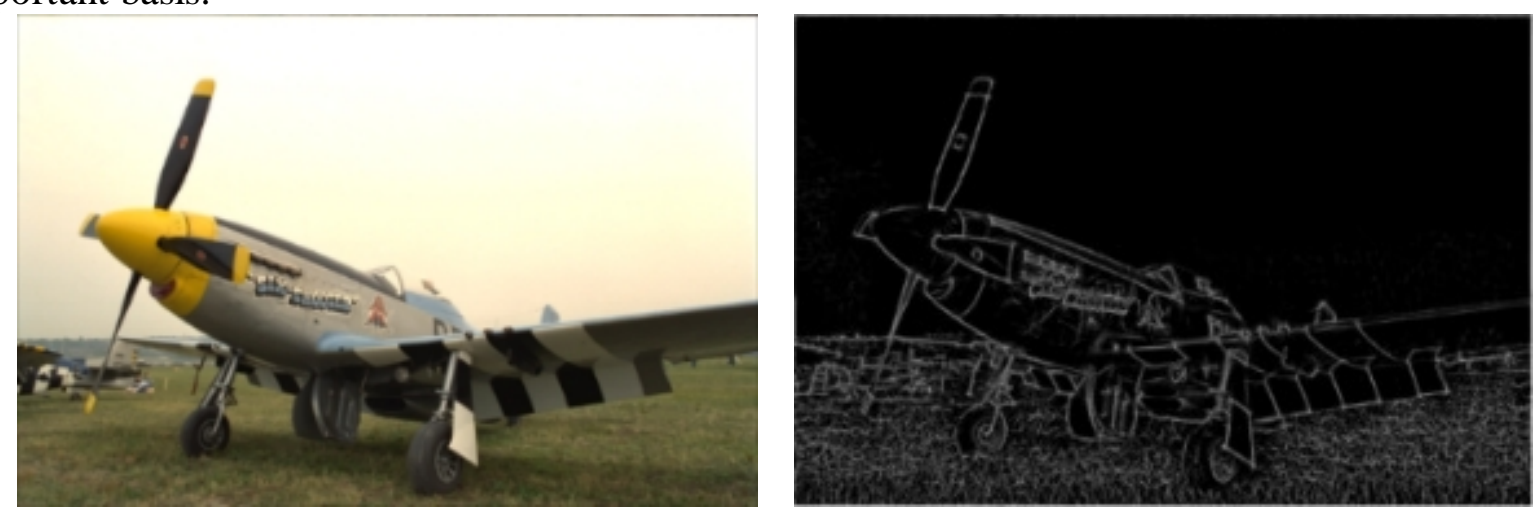

Fig.3 Original image

Fig.4 The associated phase congruency map of figure 3 

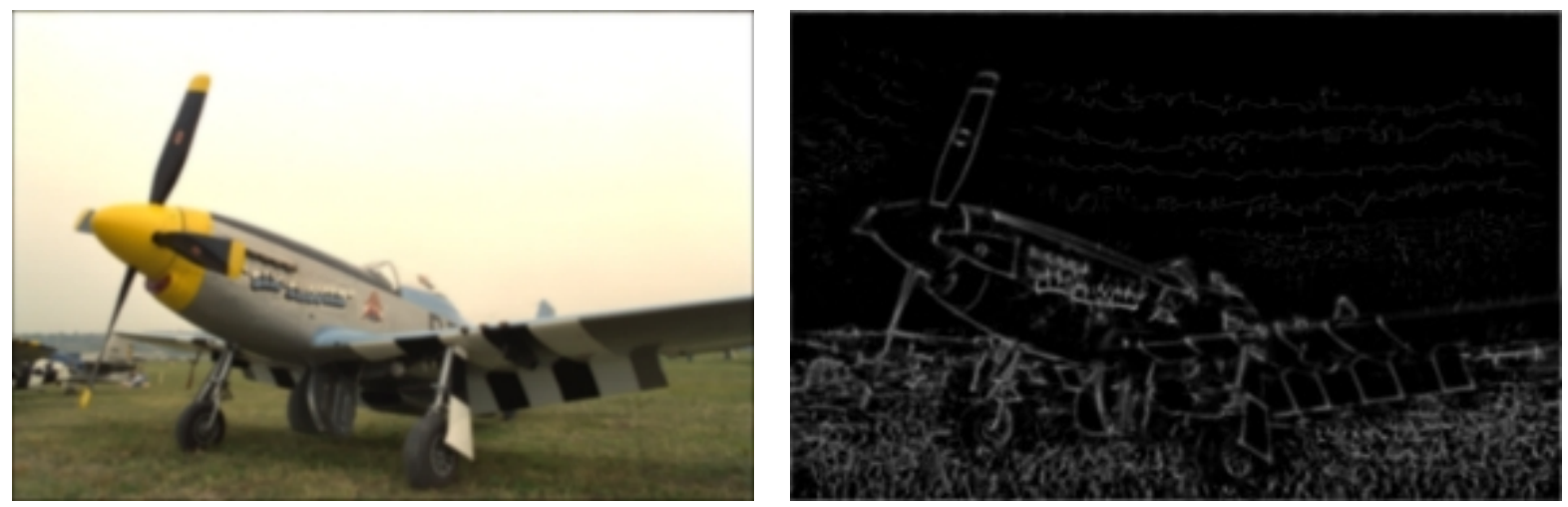

Fig.5 Blurred image

Fig. 6 The associated phase congruency map of figure 5

Gray Level Co-occurrence Matrix reflects the image texture. We extract ASM (Angular Second Moment), Entropy, contrast, homogeneity and correlation to represent the image features. ASM is the sum of the squares of the Gray Level Co-occurrence Matrix elements. So also known as energy. ASM reflects the image grey distribution uniformity degree of thickness and texture. If some values in the Gray Level Co-occurrence Matrix is big and the other value is small, the ASM is big. If all the values of Gray Level Co-occurrence Matrix are equal, the ASM is small. Entropy is a measure of the amount of image information. It represents the image of the complexity of the texture or non-uniform degree. Contrast reflects the image texture grooves of the degree of depth and clarity. Image texture grooving the deeper the contrast, the greater the visual effect is more clear. If the contrast is small, then visual effect is fuzzy. Homogeneity reflect the texture of image. Homogeneity measuring local changes of texture image. Correlation is to measure the degree of similar row or column direction of Gray Level Co-occurrence Matrix elements. Correlation size reflects the local gray correlation in the image. When the matrix element value equals the uniform, the value is big. Characteristic parameters of image values are shown in table 1 below.

Table 1 the value of the five parameters of Fig.3 to Fig.6

\begin{tabular}{|c|c|c|c|c|c|}
\hline image & ASM & Entropy & contrast & homogeneity & correlation \\
\hline Fig.3 & 0.3030 & 6.3822 & 0.1131 & 0.9600 & 0.9913 \\
\hline Fig.4 & 0.5882 & 4.5487 & 0.2821 & 0.9185 & 0.7710 \\
\hline Fig.5 & 0.3034 & 6.4241 & 0.0480 & 0.9786 & 0.9962 \\
\hline Fig.6 & 0.6145 & 4.8802 & 0.1184 & 0.9509 & 0.8553 \\
\hline
\end{tabular}

SA - GRNN model validation. This article uses the LIVE database release 2 as experiment database. The LIVE database release2 contains 174 pair of blurred image. Every image has a Difference Mean Opinion Score. In order to guarantee the correctness of the algorithm. First, we need to random grouping the 174 image. Eighty percent of the images used for training the neural network, twenty percent of the images are used to verify the accuracy of the algorithm. Due to Entropy and other characteristic value scope big difference. The numerical value of each input by dozens of times. To ensure that all data have the same importance, we need to do data normalization processing. All data values range normalization to $[0.1,0.9]$ to prevent the decimal information submerged by large number of information. Normalization formula is in the following equations (4).

$$
x_{i}=0.1+0.8 * \frac{x-x_{\min }}{x_{\max }-x_{\min }}
$$

First of all, the images were randomly assigned. In phase coincidence of the image are calculated respectively and the Gray Level Co-occurrence Matrix. Then the data normalization processing. Then use the eighty percent of image characteristics data training SA - GRNN network. After the training, use SA-GRNN to calculate the other twenty percent of the images. Calculation algorithm assessment data and the Difference Mean Opinion Score. Conclusion SROCC and CC value. Repeated 100 times, 
record the SROCC and CC 100 times. Finally, get the average value of the SROCC and CC. Image quality assessment based on SA - GRNN process are shown in figure 7 below.

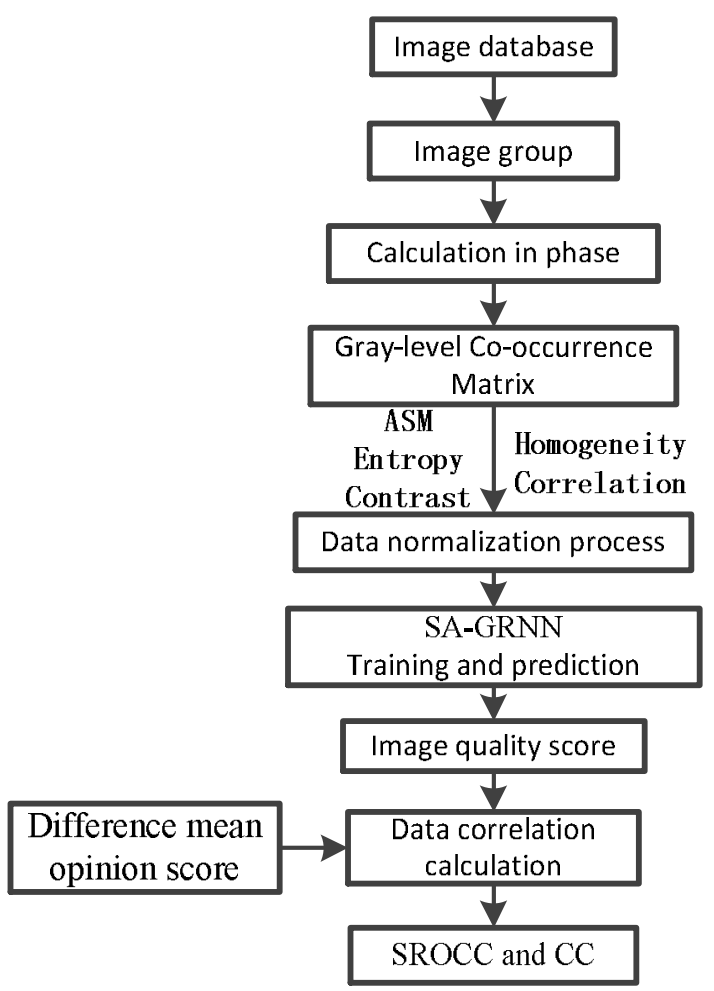

Fig.7 Flow chart of image quality assessment based on Simulated Annealing and General Regression Neural Network

After 100 times experiments, obtaining SROCC and CC average value. The experimental results of LIVE database release 2 are shown in table 2 below. The spread value is 0.15 .

Table 2 results on LIVE database 2

\begin{tabular}{|c|c|c|}
\hline & Algorithm & LIVE database 2 \\
\hline SROCC & SA - GRNN & 0.9319 \\
\hline CC & SA - GRNN & 0.9328 \\
\hline
\end{tabular}

The comparison of SA - GRNN algorithm. In order to verify the uses of algorithm in this paper. First, use the LIVE database release 2 as experiment database. Then, train the SA - GRNN model. We use the $\mathrm{CSIQ}^{[13]}$ (Categorical Image Quality Database), TID2008 ${ }^{[14,15]}$ (Tampere Image database 2008) and IVC ${ }^{[16]}$ (Image and Video Communications) for testing. Test results are shown in table 3 below. SA - GRNN algorithm on SROCC is relatively close to other algorithm and a slight lead in CC. SA - GRNN algorithm also show the generalization and applicability of the algorithm on TID2008 and IVC.

Table 3 IQA results on database (CSIQ, TID2008 \& IVC)

\begin{tabular}{|c|c|c|c|c|c|}
\hline & Algorithm & LIVE2 & CSIQ & TID2008 & IVC \\
\hline $\mathrm{S}$ & SA - GRNN & $\mathbf{0 . 9 3 1 9}$ & $\mathbf{0 . 8 9 1 8}$ & $\mathbf{0 . 6 5 1 9}$ & $\mathbf{0 . 8 8 8 4}$ \\
\cline { 2 - 6 } $\mathrm{R}$ & reference[18] & 0.9375 & 0.8963 & 0.8154 & none \\
\cline { 2 - 6 } $\mathrm{O}$ & reference [19] & 0.9352 & 0.8342 & 0.8231 & none \\
\cline { 2 - 6 } $\mathrm{C}$ & reference [20] & 0.9368 & 0.8832 & 0.8030 & none \\
\cline { 2 - 6 } $\mathrm{C}$ & reference [21] & 0.7876 & 0.7625 & 0.6667 & none \\
\hline \multirow{4}{*}{$\mathrm{C}$} & SA - GRNN & $\mathbf{0 . 9 3 2 8}$ & $\mathbf{0 . 9 2 0 2}$ & $\mathbf{0 . 6 3 8 2}$ & $\mathbf{0 . 9 2 0 7}$ \\
\cline { 2 - 6 } $\mathrm{C}$ & reference [18] & 0.8189 & 0.8572 & 0.6567 & none \\
\cline { 2 - 6 } & reference [19] & 0.9239 & 0.8934 & 0.8133 & none \\
\cline { 2 - 6 } & reference [20] & 0.9123 & 0.9056 & 0.8276 & none \\
\hline \multirow{2}{*}{} & reference [21] & 0.9478 & 0.9347 & 0.8547 & none \\
\hline
\end{tabular}




\section{Conclusion}

Test shows that, SA - GRNN has slightly improvement on accuracy and greatly improved on time performance. Based on phase image extracted angular second moment(ASM), Entropy, contrast, homogeneity and correlation characteristic parameters can describe the image feature. The experiment on separate database also show that the SA - GRNN has certain generalization and applicability. How to applied the SA - GRNN algorithm to industrial production, will be discussed in the next step.

\section{Acknowledgements}

This work was financially supported by the National Natural Science Foundation of China (11274336), Special Funds Project of Public Welfare Research \& Capacity Building in Guangdong Province in 2014: Research \& Development of IoT-based Intelligent Ubiquitous-Perceiving Key Technology for Manufacturing Execution System (Grant No. 2014A010103034).

\section{References}

[1] JANG G Y, HUANG D J, WANG X,et al, Overview on Image Quality Assessment Methods, Journal of Electronics \& Information Technology, In Chinese. 32(2010)119-226.

[2] WANG Z Y, XIAO W, No-reference digital image quality evaluation based on perceptual masking , Computer Applications, In Chinese. 26(2006)2838-2840.

[3] SHAO Y, SUN F C, LIU Y, A No-reference Image Quality Assessment Method Using Local Structure Tensor, Journal of Electronics \& Information Technology, In Chinese. 34(2012)1779-1785. [4] ZHANG Y S, CHEN Z J, JPEG Image Quality Assessment Based on Visual Sensitivity, Computer Engineering, In Chinese. 37(2011)191-193.

[5] YIN Y, No-reference blur image quality assessment based on General Regression Neural Network, LASER \& INFRARED, In Chinese. 43(2013)466-470.

[6] FENG Y R, Research and application of Simulated Annealing algorithm, KUN MING, Kunming University of Science and Technology, In Chinese,2005.

[7] JIANG L C, LIU J P, Revised Simulated Annealing Algorithm, CHINESE JOURNAL OF ENGINEERING GEOPH YSICS, In Chinese. 4(2007)135-140.

[8] M C Morrone, R A Owens, Feature detection from local energy, Pattern Recognition Lett. 6(1987)303-313.

[9] OPPENHEI MAV, LIMJS, The importance of phase in signals, Proceedings of the IEEE. 69(1981)529-541.

[10] LIU Z, LAGAN IE`RE R, Phase congruence measurement for image similarity assessment, Pattern Recognition Letters. 28(2007)166-172.

[11] H.R. Sheikh, M.F. Sabir and A.C. Bovik, A statistical evaluation of recent full reference image quality assessment algorithms, IEEE Transactions on Image Processing. 15(2006)3440-3451.

[12] Z. Wang, A.C. Bovik, H.R. Sheikh and E.P. Simoncelli, Image quality assessment: from error visibility to structural similarity, IEEE Transactions on Image Processing. 13(2004)600-612.

[13] E. C. Larson and D. M. Chandler: Most apparent distortion, full-reference image quality assessment and the role of strategy, Journal of Electronic Imaging. 19(2010)112-114.

[14] N. Ponomarenko, V. Lukin, A. Zelensky, K. Egiazarian, M. Carli, F. Battisti, TID2008-A database for Evaluation of Full-Reference Visual Quality Assessment Metrics, Advances of Modern Radio electronics. 10(2009)30-45.

[15] N. Ponomarenko, F. Battisti, K. Egiazarian, J. Astola, V. Lukin, Metrics performance comparison for color image database, Fourth international workshop on video processing and quality metrics for consumer electronics, Scottsdale, Arizona, USA. Jan. 6(2009)14-16.

[16] Alexandre Ninassi, Patrick Le Callet, Florent Autrusseau, Pseudo No Reference image quality metric using perceptual data hiding, Human Vision and Electronic Imaging, San Jose, CA, USA, January. 6057(2006)124-130. 